\title{
OBSERVATION OF THE LINEAR POLARIZATION OF PULSAR INTEGRATED PULSES AND SUB-PULSES AT METER WAVELENGTHS
}

\author{
S. A. Suleymanova And V. D. Pugachev \\ Radio Astronomy Department, Lebedev Physical Institute
}

\begin{abstract}
A brief review of polarization observations at 103,60 , and $40 \mathrm{MHz}$ is given. Our peculiar measurement technique allows us to obtain average polarization profiles as well as statistical distributions of the polarization parameters of individual pulses and subpulses. Some examples are given in this paper.
\end{abstract}

\section{Measurement technique}

Since the early 1980 's meter-wavelength polarization measurements of pulsars have been carried out using the large BSA (103 MHz) and DKR-1000 $(60,40 \mathrm{MHz})$ telescopes. Since the telescopes are linearly polarized antennas, a special technique is used, based on the Faraday rotation of the linear polarization plane in the magnetized interstellar plasma. Faraday rotation produces a frequencydependent quasi-sinusoidal intensity modulation at the output of a multichannel radiometer. For observations at $103 \mathrm{MHz}$ we usually used a radiometer with $64 \times 20-\mathrm{kHz}$ contiguous channels. At 60 and $40 \mathrm{MHz}$ a radiometer with $32 \times 5-\mathrm{kHz}$ channels with a total bandwidth $160 \mathrm{kHz}$ was used.

Every sample in pulse longitude has its own phase and amplitude within the overall sinusoidal spectrum. These spectra were fitted to the equation

$$
a_{k}^{i}=a^{i} \sin \left(2 \pi / \Delta F f_{k}+\psi^{i}\right)+b^{i},
$$

where $i$ is the longitude of the sample, $f$ the frequency of the $k^{\text {th }}$-channel, $a^{i}, \psi^{i}$, and $b^{i}$ the parameters which define the polarization characteristicsthat is, $P_{\text {in }}=a^{i} / b^{i}[\%]$, and the position angle $P A=\psi^{i}$. The period of the Faraday modulation $-\Delta F=17.48 f_{\mathrm{c}}^{3} / R M(R M$ is the rotation measure for a given pulsar) was calculated for the central frequency of the radiometer and fixed for all samples along the pulse. As the time resolution was typically several milliseconds, we obtained well defined profiles in total intensity, fractional linear polarization and position angle for average profiles and for individual pulses (subpulses).

\section{Average profile polarization}

The average profile for a given pulsar is the result of averaging hundreds of individual pulses synchronously with the pulsar period. The polarization characteristics of the average profile were measured

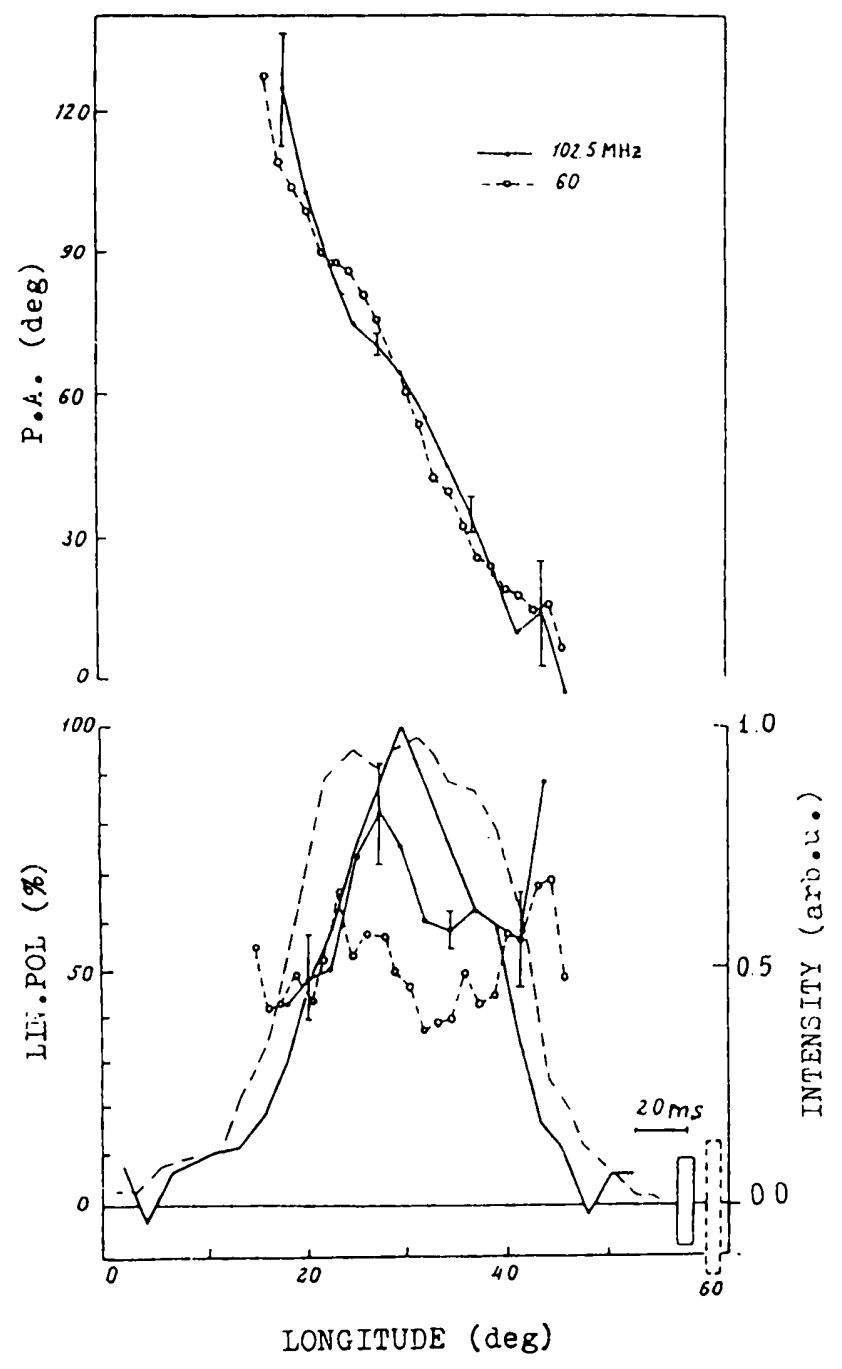

Figure 1 Average polarization profiles for PSR 0628-28 at 102.5 (solid lines) and $60 \mathrm{MHz}$ (dashed lines); the rms baseline noise and the sample intervals are shown by the resolution boxes.

for 18 pulsars at $103 \mathrm{MHz}$ and for 9 pulsars at 60 and/or $40 \mathrm{MHz}$. These results were published recently by Suleymanova (1989).

The profiles for PSR 0628-28 in figure 1 illustrate the fact that measurements made at different frequencies, using different antennas and radiome- 


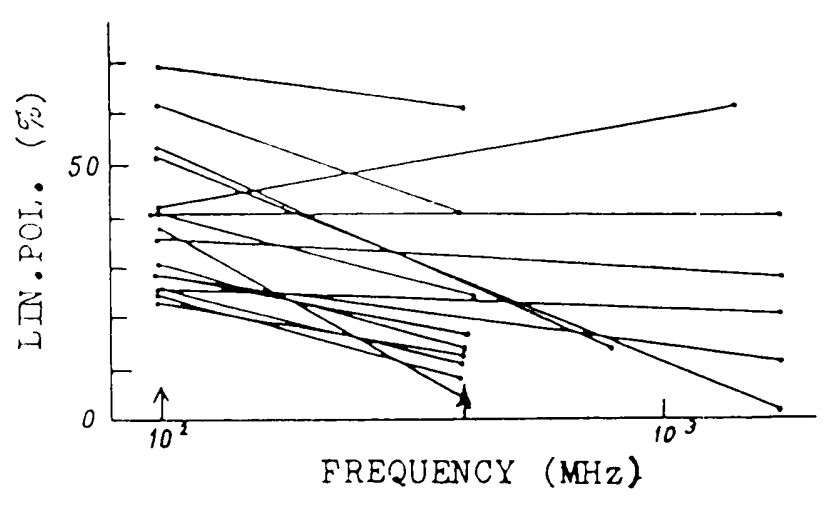

Figure 2 Frequency dependence of the linear polarization of the average profile for 18 pulsars between $100 \mathrm{M} \mathrm{Hz}$ and $400 \mathrm{M} \mathrm{Hz}$ (schematically). Observations at higher frequencies were used when data at $400 \mathrm{MHz}$ were unavailable.

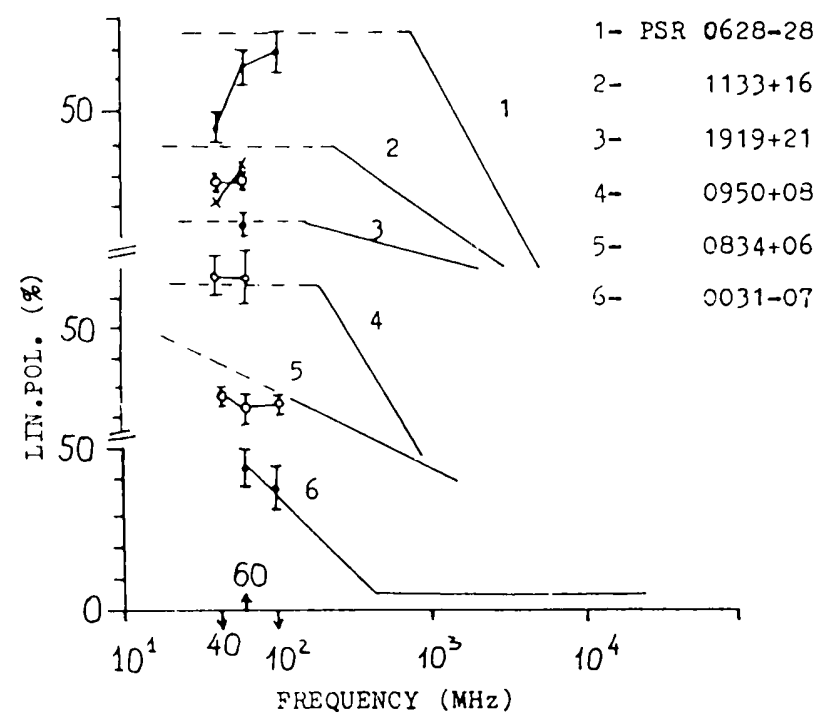

Figure 3 Linear polarization data for the strongest pulsars at 103,60 and $40 \mathrm{M} \mathrm{Hz}$. Percentage behavior at higher frequencies is shown schematically. Dashed lines show the behavior predicted by Manchester et al. (1973). For PSR $1133+16$ simultaneous observations are indicated by an " $x$ ".

ters, yield very similar results. This similarity supports the validity of our measurement technique.

It is of great interest to know how the fractional linear polarization $P_{\text {in }}$ of profiles behaves at low frequencies. Observational results are as follows:

1. The linear polarization at $103 \mathrm{MHz}$ is generally higher than at $400 \mathrm{MHz}$ (see figure 2).

2 . Below $103 \mathrm{MHz}$ (see figure 3 ) $P_{\text {in }}$ can increase (as for PSR 0031-07) or be constant as was predicted earlier (Manchester, Taylor, and Huguenin 1973) for pulsars $0950+08$ and $1919+21$.

3. Additionally, two pulsars, PSR 0628-28 and PSR $1133+16$, show polarization "turn-off" at low frequencies. Such a "turn-off" is confirmed by observations of PSR $1133+16$ simultaneously at 60 and $40 \mathrm{MHz}$.

This results in a decrease of $P_{\text {in }}$ in the sec-

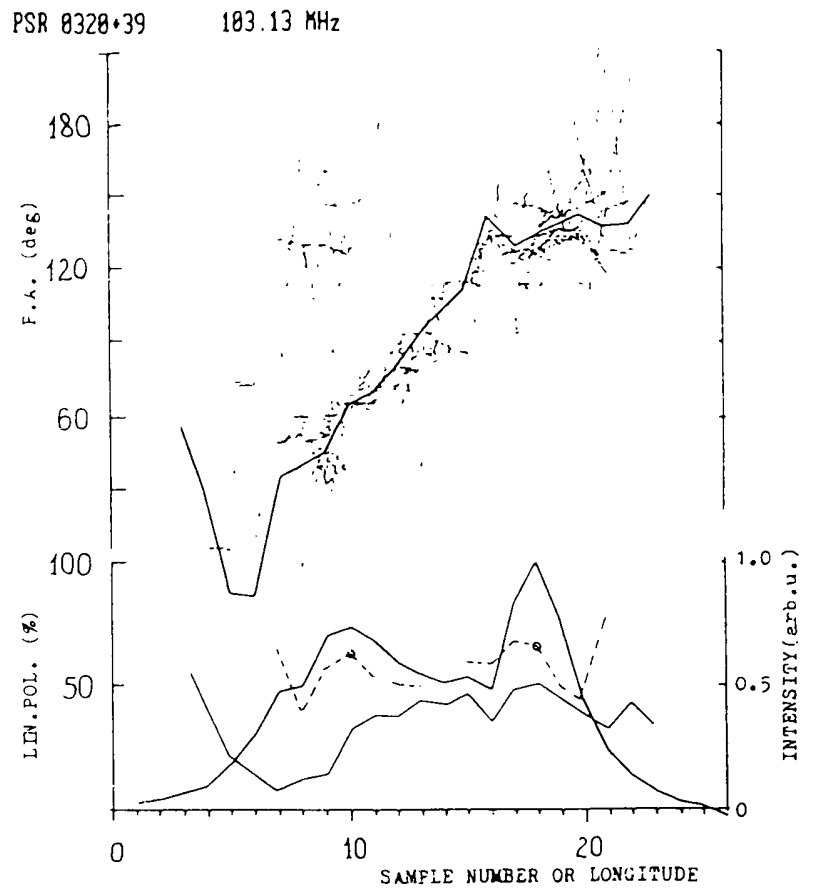

Figure 4 Average polarization profiles of PSR 0320+39 at $103 \mathrm{MHz}$. Statistical distributions of the position angle for individual pulses and the mean linear polarization profile for subpulses in the first and second components (dashed lines) are shown. The sample interval is $5.184 \mathrm{~ms}$.
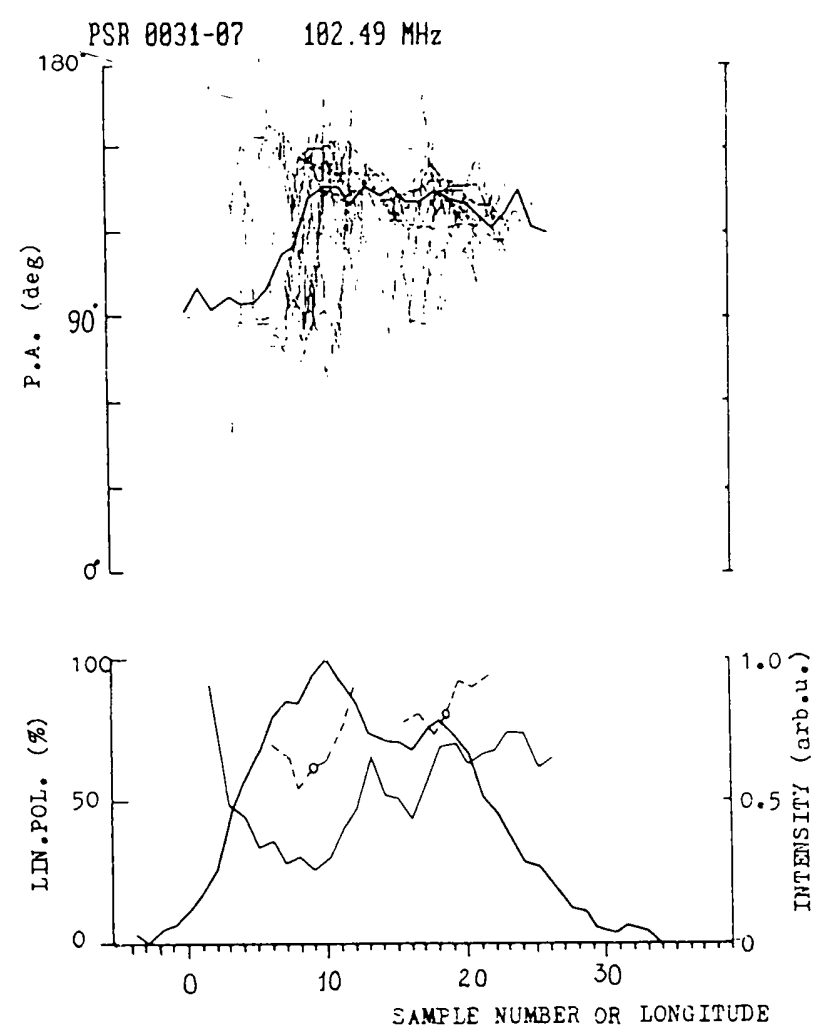

Figure 5 PSR 0031-07 (see figure 4). The sample inter$\mathrm{val}$ is $5.184 \mathrm{~ms}$.

ond, more intensive component of the pulsar below $100 \mathrm{MHz}$. The statistical distribution of the $P A$ for individual pulsars shows that quasiorthogonally polarized radiation spans both compo- 


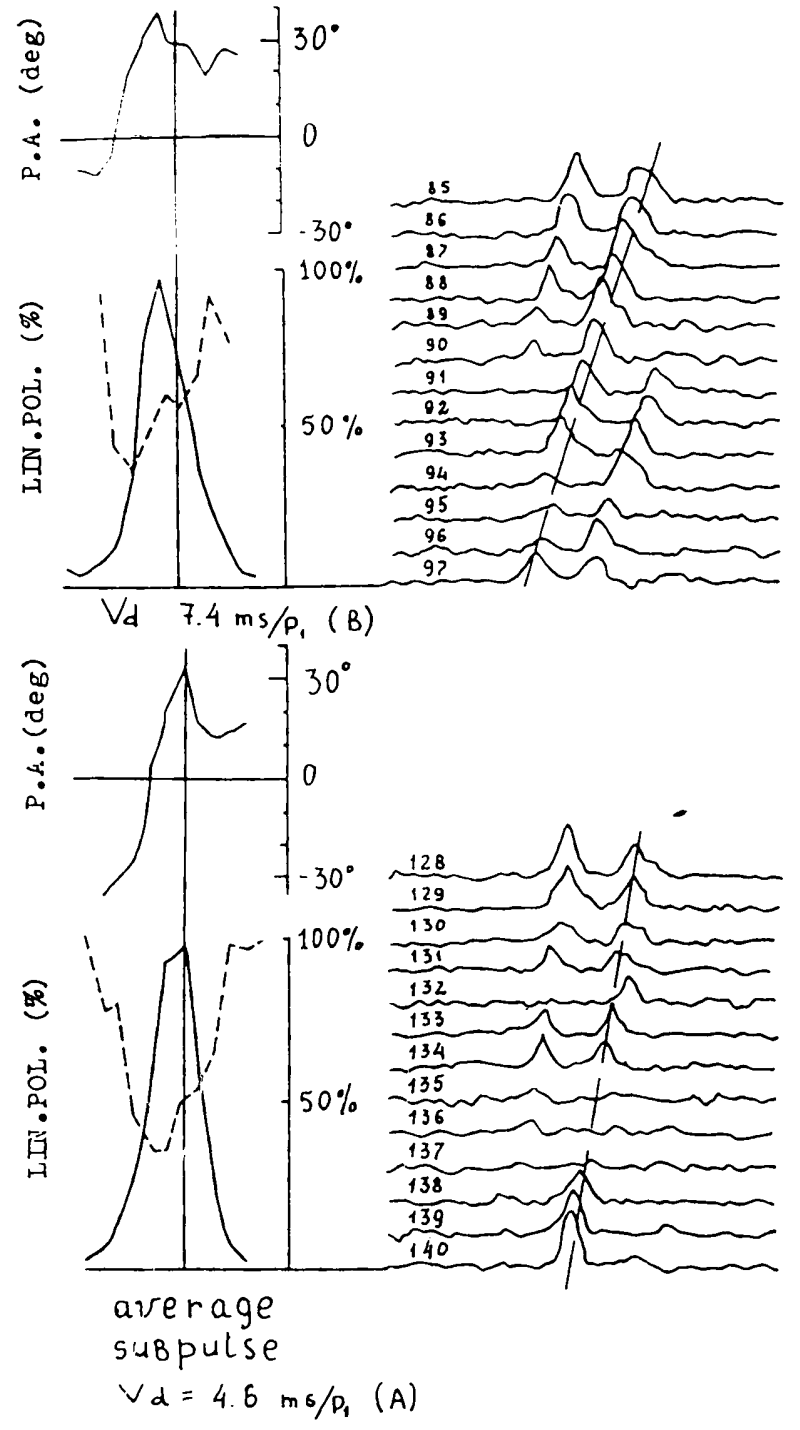

Figure 6 Two sets of consecutive pulses of PSR 0031-07 at $103 \mathrm{MHz}$, drifting with different rates $B$ and $A$. When averaged along a drift band they form very similar average subpulse polarization profiles.

nents of PSR $1133+16$, while at higher frequencies it occurs only in component one.

\section{Drifting subpulse polarization}

Pulsars 0031-07 and $0320+39$ are known to exhibit very regular subpulse drift behavior. While the subpulses of PSR $0320+39$ drift "abnormally" from the leading to the trailing edge of the average profile, the subpulses of PSR 0031-07 drift in the opposite ("normal") direction. PSR 0031-07 is also known to switch between three harmonically related drift rates $\mathrm{A}, \mathrm{B}$ and $\mathrm{C}$ (Huguenin, Taylor, and Troland 1970). So, we had the possibility of investigating the subpulse polarization behavior as a function of drift rate and direction.

The results are as follows:

1. For both pulsars, independent of the drift
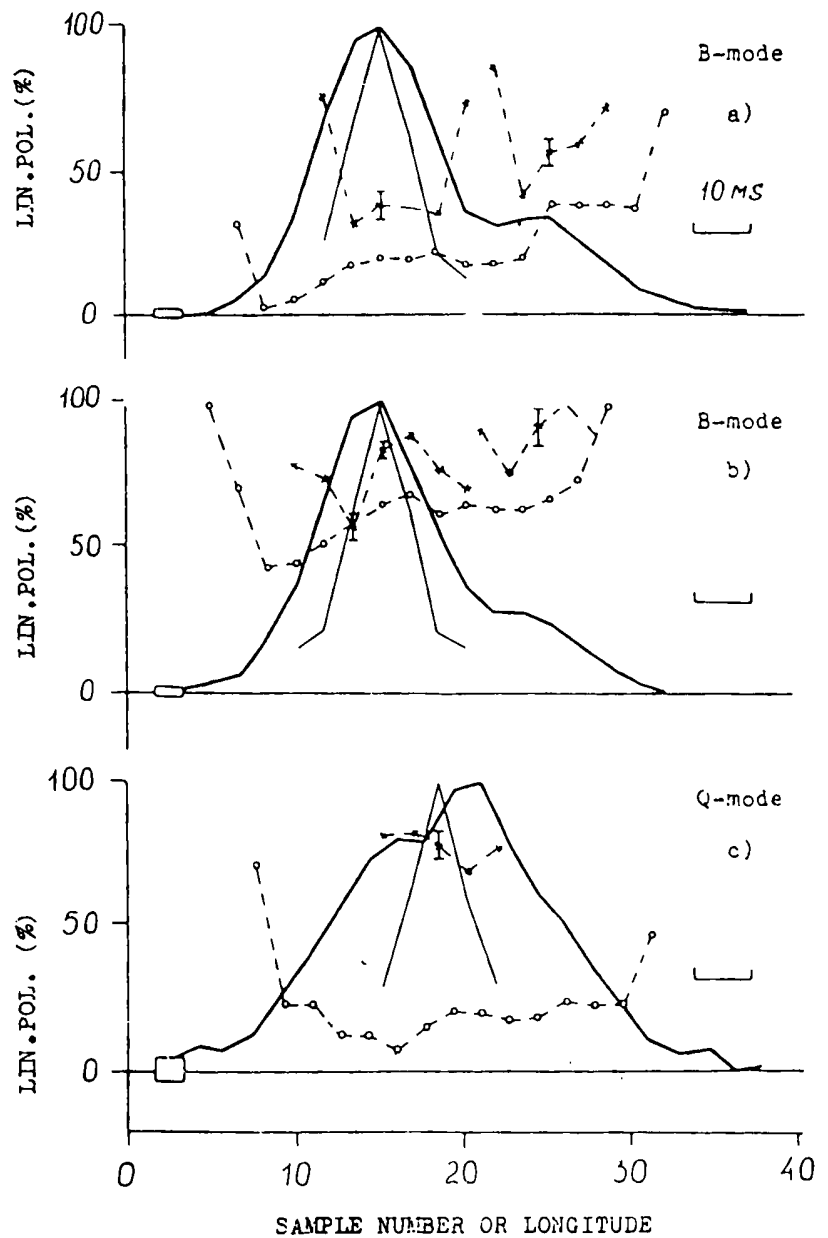

Figure 7 Three 200-pulse averages of PSR 0943+10 together with $R_{\text {in }}$ profiles ( $\left.-\circ\right)$. Subpulse polarization profiles were measured and then averaged after combination subpulse maxima (-*-).

direction, a deep minimum of $P_{\text {in }}$ is evident for the first component of the double-shaped average intensity profile. Depolarization is caused by the increasing numbers of $P A$ jumps in this longitude region (see figures 4 and 5).

2. For PSR 0031-07 the polarization profiles of the subpulses, averaged along a drift band for drift rates $A$ and $B$, look very similar (figure 6 ) and resemble the $403-\mathrm{MHz}$ profiles of Manchester, Taylor, and Huguenin (1975). We can conclude that the polarization properties of subpulses are poorly correlated with their drift parameters.

\section{Time variations in the polariza- tion state of average pulses and subpulses}

PSR 0943+10 is known to be a "mode-switcher" (Suleymanova and Izvekova 1984). It has two distinct average profiles: the more intense doubleshaped B-mode and the weaker single (or double- 
unresolved?) Q-mode profile. Routine day-to-day observations have shown significant changes in the polarization state for both modes. Variations of the fractional linear polarization of the average profile and subpulses can correlate (as in figure $7 \mathrm{a}$ and $\mathrm{b}$ ) or not (see figure $7 \mathrm{c}$ ). In the latter case (c), the generally highly polarized subpulses result in depolarization of the average profile due to strong pulse-topulse position-angle variations. For case (b) these variations are moderate. This leads us to the conclusion that pulse-to-pulse variations can change drastically with a time scale of days or hours. The character of the statistical distribution of $P A$ (not shown here) confirms this idea.
Another important conclusion from our point of view is that the subpulse polarization can either be sensitive to orthogonally polarized radiation (see (figure $7 a$ and $b$ ) or not (figure 7c). The two orthogonally polarized radiation intensities compete inside the subpulse. In case (c) only one polarization mode is present; therefore the polarization of the subpulse is high. Increasing the influence of orthogonally polarized radiation inside the subpulse causes depolarization of the leading part of the subpulse [case (b)] and then depolarization of the whole subpulse. The character of the competition with the observed depolarization, varying from day to day, has yet to be investigated. 